\title{
Investigating the Evolution of Cooperative Behaviour in a Minimally Spatial Model
}

\author{
Simon T. Powers and Richard A. Watson
}

School of Electronics and Computer Science, University of Southampton, Southampton, U.K., SO17 1BJ; stp05r@ecs.soton.ac.uk; raw@ecs.soton.ac.uk

\begin{abstract}
It is well known that the evolution of cooperative behaviour is dependant upon certain environmental conditions. One such condition that has been extensively studied is the use of a spatially structured population, whereby cooperation is favoured by a reduced number of interactions between cooperators and selfish cheaters. However, models that address the role of spatial structure typically use an individual-based approach, which can make analysis unnecessarily complicated. By contrast, non-spatial population genetics models usually consist entirely of a set of replicator equations, thereby simplifying analysis. Unfortunately, these models cannot traditionally be used to take account of spatial structure, since they assume that interaction between any pair of individuals in a population is equally likely. In this paper, we construct as model that is still based on replicator equations, but where spatial localisation with respect to the number of interactions between individuals is incorporated. Using this model, we are able to successfully reproduce the dynamics seen in more complex individual-based models.
\end{abstract}

\section{Introduction}

It is well known that the existence of spatial structure in a population can (although not always, e.g. [1]) facilitate the evolution of cooperative strategies. For example, consider an evolving population of individuals playing the Prisoner's Dilemma game, where the payoff that an individual receives modifies its fitness. If an individual plays only one round against each opponent, and the opponents are drawn at random, then a population initialised with a mixture of cooperate and defect strategies will evolve towards every individual playing defect [2]. This situation corresponds to a freely-mixed world with no spatial structure, since an individual is equally likely to interact with any other member of the population. On the other hand, if the population has a spatial structure, so that an individual only plays against its neighbours, then it is possible for spatial clusters of cooperators to persist [3]. This is due to the fact that for the cooperate strategy to be viable, the cooperator must play against other cooperators and minimise contact with defectors.

These two cases of freely-mixed and spatial worlds are often modelled using different techniques. To model the evolution of the population in the freely-mixed case, it is only necessary to keep track of the proportion of individuals playing 
each strategy. How the frequency of each strategy changes over time can then be calculated by means of differential or difference equations; this is known as a replicator dynamics model [4]. By contrast, the modelling of the evolution of a spatially structured population is usually achieved by explicitly representing each individual; this technique is known as individual-based modelling. An example of such a model is that of the spatial Prisoner's Dilemma produced by Nowak \& May [3], where a rectangular grid was divided into cells, with each cell containing one individual. In this model, interactions only occurred between neighbouring cells.

The problem with using an individual-based model is that it can be a difficult and lengthy task to understand the root processes causing the observed behaviour [5]. For example, Nowak \& May [3,6] found that chaotic patterns of cooperate and defect cells emerged in their apparently simple 2D grid model. Conversely, replicator dynamics models are easy to analyse due to their transparency; the processes at work are explicitly stated in the difference equations. However, they cannot be used in the spatial case because they assume that each individual is equally likely to interact with any other individual. In this paper we aim to present a model that combines the simplicity of replicator equations with the ability of individual-based approaches to model spatially-structured populations.

Our model is based on the scenario of different growth (replication) rates under resource-limited conditions. Specifically, we assume that a trade-off exists between growth rate and efficiency of resource usage, an idea that has been widely explored in biological models. Specific examples of existing models that address this trade-off include individual-based models of bacterial growth [7-9], and models of the virulence versus productivity trade-off in disease organisms $[10,11]$. Such models usually consider two strategies; a low growth rate / high efficiency strategy (cooperative), and a high growth rate / low efficiency strategy (selfish). If none of the limiting resource is shared between individuals playing different strategies, then the cooperative type will win due to its efficiency advantage. Conversely, if the resource is freely-shared between different strategies, then the selfish type will win, due to its larger growth rate [7-9]. This is despite the fact that the wasteful resource usage of the selfish type means that it will reach a smaller biomass than if all individuals used the cooperative strategy, a scenario known as the "Tragedy of the Commons" [12].

We model groups of individuals using the same growth rate / efficiency strategy as indivisible "colonies", where the ratio of colony biomasses (sizes) can be interpreted as strategy frequencies. A colony therefore represents a particular growth rate strategy. By treating all individuals with the same strategy / genotype uniformly, our model is still able to use replicator equations. This amounts to an assumption that spatial structure within a colony does not matter with respect to the result of competition with another colony, i.e. that the effect of spatial localisation between individuals playing the same strategy is insignificant. A key aim of this paper is to show that given this assumption, the results with respect to the evolution of cooperation are unchanged compared to those 
models that do explicitly model spatial structure between individuals playing the same strategy.

Spatial localisation in interactions between strategies / colonies is introduced by providing each colony with its own resource supply. The degree of spatial localisation can then varied by changing the amount of this resource that is shared with the other colony. This approach can therefore model completely spatial and freely-mixed worlds, as well as positions between these extremes.

The key concept of spatially structured populations that is relevant to the evolution of cooperation is a reduced frequency of interactions with

non-neighbours. By introducing degree of spatial localisation as degree of resource segregation, we aim to capture this key concept. We then hypothesise that this is all that is necessary to capture the competition and invadability dynamics seen in more complex models.

The advantage of this minimal approach is that it allows our model to contain fewer parameters and assumptions compared to those that use an individualbased approach. For example, the authors of grid-world style individual-based models must make decisions such as to only allow an individual to reproduce if there is an empty cell next to it, as was the case in [8]. It is then not clear whether this decision will affect the results obtained. The aim of our minimally spatial model is to include only the concept of space in a population which affects the evolution of cooperation, namely reduced interactions with non-neighbours. Consequently, the number of modelling decisions to be made are minimised. Furthermore, the use of replicator equations eases analysis by making explicit the processes at work.

\section{A Minimal Model of Colony Growth}

A definition of the symbols used throughout this paper is provided in Table 1.

The change in biomass of a colony at each time-step in our model is as stated in $(1)$.

$$
b_{i(t+1)}=b_{i(t)}+g_{i(t)}-k_{i(t)}
$$

This shows that the biomass of a colony changes due to both growth and death. The process of colony growth is then as defined in (2). Specifically, the magnitude of biomass increase made by a colony is proportional to both its biomass and to the amount of available resource, where the constant of proportionality is its intrinsic growth rate, as defined by its strategy. The amount of growth a colony can make is therefore limited by the amount of resource that is available to it.

$$
g_{i(t)}=G_{i} b_{i(t)} r_{i(t)}
$$

The amount of mortality suffered by the colony is given in (3), and is proportional to biomass and inversely proportional to resource availability.

$$
k_{i(t)}=\frac{K b_{i(t)}}{r_{i(t)}+1}
$$


Table 1. Definition of symbols.

\begin{tabular}{|l|l|}
\hline Symbol & Definition \\
\hline$G$ & Growth rate constant \\
\hline$U$ & Resource usage rate constant \\
\hline$I$ & Resource influx constant \\
\hline$K$ & Death rate constant \\
\hline$D$ & Resource diffusibility constant \\
\hline$b$ & Biomass \\
\hline$g$ & Growth achieved at a given time-step \\
\hline$k$ & Death at given time-step \\
\hline$r$ & Available resource level \\
\hline$c$ & Resource consumed at given time-step \\
\hline$d$ & Magnitude of resource diffusion \\
\hline$\beta$ & Modification of $d$ based on relative colony biomasses \\
\hline$M$ & Maximum effect that $\beta$ can have \\
\hline$i$ & Colony index \\
\hline$t$ & Time index \\
\hline \hline
\end{tabular}

The amount of resource consumed by the colony at a time-step (4) is proportional to its growth, with its intrinsic resource usage rate, defined by its strategy, as the constant of proportionality.

$$
c_{i(t)}=U_{i} g_{i(t)}
$$

The net change in a colony's resource level depends upon three things (5): the resource used during growth (4), a constant influx from the environment to the colony, and exchange of resource with a competing colony via diffusion (6).

$$
\begin{aligned}
& r_{i(t+1)}=r_{i(t)}+I-c_{i(t)}+d_{i(t)} \\
& d_{i(t)}=D\left(\beta_{j(t)} r_{j(t)}-\beta_{i(t)} r_{i(t)}\right)
\end{aligned}
$$

The diffusion process in (6) acts to try and equilibrate the resource levels of two competing colonies, $i$ and $j$. How large an effect this has depends on two things: The setting of the resource diffusibility constant and a term $\beta$, defined in (7) and (8) below. Changing the diffusibility constant has the effect of varying the degree of spatial localisation; if it is set to 0.5 , then each colony's resource supply is shared freely with the other colony. On the other hand, if it is set to 0 then no movement of resource (interaction) between colonies occurs.

$$
\begin{gathered}
\beta_{i(t)}=1, \text { if } b_{i(t)}>b_{j(t)} \\
\beta_{i(t)}=1+(M-1)\left(\frac{1}{b_{i(t)}}\right), \text { otherwise }
\end{gathered}
$$

The role of $\beta$, as defined in (7) and (8), is to make the magnitude of the force of diffusion dependant upon the respective colonies' biomasses. This is motivated by the idea of outward geometric colony growth from a single point in a 
spatial world. Specifically, in a small colony, a greater proportion of the colony members will be on the colony's edge and therefore in contact with members of the competing colony. By contrast, in a larger colony, a greater proportion of the colony's members will be shielded from such contact by the layer of edge members. Therefore, small colonies will have a greater proportion of their members interact with the rival colony, hence providing the motivation for increased resource sharing if a colony is small. The purpose of (7) is to make this change operate in one direction only, i.e. so that the amount of resource transferred via diffusion can only ever be increased by this effect. $M$ is a constant that determines the maximum effect that this process can have.

Finally, it is worth stressing that movement of resource only occurs between, and not within, colonies. Within a colony, all individuals are treated in a nondifferentiated manner. This factor allows colony growth to be modelled via the replicator equation (1).

\section{Investigations with the Model}

This section details three scientific questions that can be addressed with the model, presenting detailed results of our investigations into the first two. In all of the experiments, we consider two competing growth rate / resource efficiency strategies. Namely, a cooperative strategy that uses resource efficiently but that grows at a slower rate, and a selfish strategy that grows at a faster rate but that wastes the growth-limiting resource.

It should be noted that we do not, in these experiments, introduce new strategies via mutation. Instead, we explore how strategy frequencies change through time, in the style of evolutionary game theory [13]. Finally, throughout this paper, the setting of the model constants given in Table 2 are used.

Table 2. Settings of model constants used throughout the paper.

\begin{tabular}{|l|l|}
\hline Constant & Value \\
\hline$G_{\text {selfish }}$ & 0.02 \\
\hline$G_{\text {cooperative }}$ & 0.01 \\
\hline$U_{\text {selfish }}$ & 0.2 \\
\hline$U_{\text {cooperative }}$ & 0.1 \\
\hline$I$ & 1 \\
\hline$K$ & 0.1 \\
\hline$M$ & 2 \\
\hline
\end{tabular}

\subsection{Equilibrium Size of Cooperative and Selfish Colonies under Varying Degrees of Spatial Localisation}

The most obvious question that can be addressed with the model is how the equilibrium size of competing colonies of cooperative and selfish individuals varies 


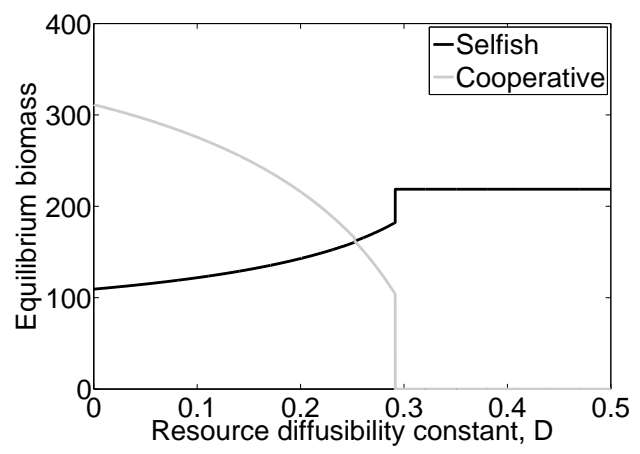

Fig. 1. Equilibrium biomasses of cooperative and selfish colonies.

as the degree of spatial localisation changes. This question can be addressed through our model by starting each colony off with unit biomass and iterating through the difference equations until the equilibrium biomasses are reached. The results of this, for varying $D$, are presented in Fig. 1 .

These results successfully reproduce those found in more complex models. Specifically, in a freely-mixed world (large $D$ ) the slower growing cooperators are driven extinct by their less efficient but faster growing rivals, as in the "Tragedy of the Commons" [12]. Conversely, under maximal spatial localisation coexistence of both types is obtained, with the cooperators reaching a larger biomass. Therefore, our model has successfully captured the relevant concepts of spatial localisation, without recourse to individual-based modelling.

What happens between the extremes of freely-mixed and maximal spatial localisation cannot be predicted directly from the equations. In particular, an interesting finding is that as soon as $D$ moves above a critical threshold, the cooperative colony is driven to extinction. The reason for this sudden extinction is that when the cooperative colony's biomass drops below a critical size then it looses too much resource to the selfish colony, due to the $\beta$ term defined in (8). The loss of a large amount of resource means that the cooperative colony can no longer maintain its biomass level, i.e. its biomass loss due to mortality is greater than its biomass increase due to growth. This then sets off a positive feedback loop whereby as the cooperative colony's biomass decreases it looses more resource to the selfish colony, which then causes its biomass to drop even further and so on. It is the initiation of this positive feedback loop that causes the cooperative colony to go extinct as soon as $D$ rises above the threshold.

\subsection{Invadability Dynamics}

Our model can also be used to investigate issues of strategy invadability [13]. Means by which an invader playing another strategy can arise in nature include genetic mutation [11,14], phenotypic switching of individuals due to changing environmental conditions [9] and migration of individuals from other demes [15]. 
Invasion scenarios are created in our model by allowing a colony of one strategy to grow to equilibrium biomass, and then introducing a colony of a different strategy into the same world. A successful invasion is one in which the invading colony survives above 0 biomass and therefore moves the strategy frequencies to a new equilibrium. On the other hand, if the invasion fails then the strategy frequencies will remain at their previous equilibrium, i.e. where the host strategy is at fixation.

The invadability dynamics present in existing models that we aimed to recreate are, firstly, that a single selfish mutant should always be able to invade into a cooperative host. In other words, a colony of cooperators should always be vulnerable to exploitation by a selfish cheater that grows at a faster rate. The second dynamic seen in other models is that cooperators can only invade into a selfish host if there is a sufficient number of them, so that the majority of their interactions will be with each other, rather than with the host. This idea of there being fewer interactions with the host in a larger invading colony is captured in our model by the fact that the amount of resource exchanged between host and invader via diffusion is inversely proportional to the biomass of the invader, as per (8). We therefore hypothesised that all that is required to reproduce this dynamic is to make the amount of resource shared / number of interactions between host and invader inversely proportional to the number of invaders.

We first considered the vulnerability to invasion of a cooperative host by a selfish invader. The results obtained were that an invading selfish colony of unit biomass could always survive and grow, regardless of the degree of spatial localisation. The equilibrium strategy frequencies reached after an invasion under varying degrees of spatial localisation are shown in Fig. 2. The key trend is that the frequency of the selfish strategy increases with $D$, as would be expected from the results in section 3.1. Furthermore, at settings of $D$ above approximately 0.32 the cooperative host is driven to extinction by the growth of the invader. The conclusion is that a coexistence of cooperative host and selfish invading strategies is reached in worlds with moderate degrees of spatial localisation $(D<0.32)$, while the invading selfish strategy reaches fixation in more freely-mixed worlds $(D \geq 0.32)$.

The second question that we consider is the size of a cooperative colony necessary to invade a selfish host. Figure 3 shows the necessary size under varying degrees of spatial localisation, where the necessary biomasses for invasion have been rounded to the nearest integer. Below a threshold value of $D=0.17$, invasion by unit biomass is always successfully. Above this point, but below a second threshold, cooperative colonies with larger biomasses can successfully invade. Such colonies of greater than unit biomass are analogous to clusters in grid-world style models. The advantage of clusters of cooperators comes from reduced interactions with the selfish host. Our model has shown that this can be achieved simply by making the amount of resource shared inversely proportional to the invader's biomass. 


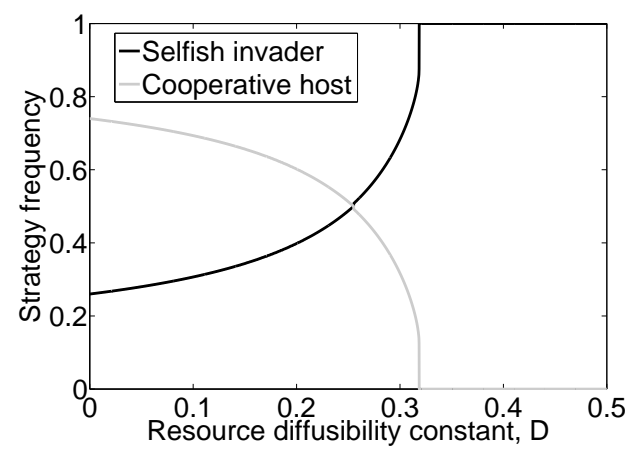

Fig. 2. Equilibrium strategy frequencies after invasion of a cooperative host by a selfish colony, under varying degrees of spatial localisation, $D$.

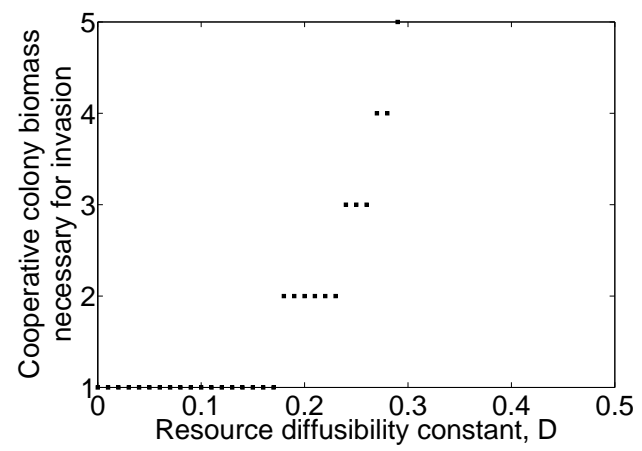

Fig. 3. Biomass of a cooperative colony necessary to invade a selfish host, under varying degrees of spatial localisation, $D$. Invasion is not possible for values of $D$ above 0.28 .

\subsection{Genetic Mutation as a Means of Adaptively Setting the Growth Rate / Efficiency Trade-Off}

The previous section considered invadability dynamics where the invading strategy was very different from that of the host, i.e. there was a large difference in their growth rate / efficiency parameter setting. However, the strategy space in our model is continuous, allowing for a small change in strategy to arise, e.g. via genetic mutation. It may therefore be possible for the canonical cooperative strategy used throughout this paper to evolve through a series of successive small mutations, where each mutant is slightly less selfish than the host strategy. Of most significance is the fact that this may be able to occur in worlds where the canonical cooperative type cannot invade directly as an individual (colony of unit biomass).

We therefore address in this section the question of whether or not a single mutant (colony of unit biomass) that is only slightly more cooperative than the established selfish strategy can successfully invade. We hypothesise that this only 
slightly more cooperative mutant will be able to invade at settings of $D$ where a more cooperative type cannot. The reasoning is that the closer the mutant is to the established selfish type, the faster it will grow and prevent a large resource flow away from itself via (8).

To test this hypothesis, we considered a world occupied by a single selfish colony at equilibrium biomass and where $D=0.24$. At this setting, an initial biomass of 3 is necessary for the cooperative strategy to invade a host selfish colony (see Fig. 3). However, we considered a strategy whose growth and resource usage rates are both $95 \%$ of that of the selfish type, i.e. only slightly more cooperative. We found that unit biomass of this strategy could invade a host selfish colony, with equilibrium strategy frequencies of 0.49 for the host and 0.51 for the invader. This successful invasion by unit biomass of a slightly more cooperative type suggests that the canonical cooperative type could arise via a series of small mutations in worlds where it cannot invade directly.

\section{Conclusion}

It is well known from a plethora of existing individual-based models that a spatially structured population can promote cooperative behaviour. In this paper, we have presented a very simple model, based on replicator equations, that allows us to reproduce key results from these more complex models. In particular, we have chosen to capture the concept of spatial localisation in interactions between individuals using the concept of degree of resource sharing. Although our model is framed in terms of colony growth, the questions that it addresses are much more general and are fundamental to explaining the evolution of cooperative behaviour.

The first key conclusion from our model is that, in order to capture the effects of spatial localisation on the evolution of cooperation, it is only necessary to model the effect of spatial localisation between individuals playing different strategies; individuals playing the same strategy can be treated homogeneously. The second key conclusion is that the invadability results from individual-based spatial models, in which cooperators can usually only invade in clusters, can be reproduced simply by making the amount of resource shared inversely proportional to the invader's biomass. This therefore captures the notion of a spatial cluster of cooperators, without having to use a more complex grid-world style model or a social network [16].

It is unavoidable that choices are made about the specific details of the equations defining the model, and of the settings of the constants. It follows that changing these details would quantitatively change the results, e.g. the values of $D$ for which strategy coexistence is sustainable and the colony sizes necessary for invasion. However, qualitative properties of the dynamics should be robust, i.e. there should always be a threshold value of $D$ below which strategy coexistence is sustainable. Furthermore, there were less choices to be made with our approach than with an individual-based model. From this perspective, our results are more robust than those of individual-based models, since there are less parameters to 
be set. On the other hand, our model is more general than traditional replicator dynamics approaches, as we have been able to model spatial localisation, something that cannot be done with a traditional replicator dynamics model.

In future work, we intend to investigate the relationship between our model and spatial and freely-mixed versions of the Prisoner's Dilemma game. In particular, we would like to make explicit the relationship between the cooperative and selfish strategies of colony growth in our model and the cooperate and defect strategies in the Prisoner's Dilemma.

\section{References}

1. Hauert, C., Doebeli, M.: Spatial structure often inhibits the evolution of cooperation in the snowdrift game. Nature 428 (2004) 643-646

2. Axelrod, R., Hamilton, W.D.: The evolution of cooperation. Science 211(4489) (1981) 1390-1396

3. Nowak, M.A., May, R.M.: Evolutionary games and spatial chaos. Nature 359 (1992) 826-829

4. Taylor, P.D., Jonker, L.B.: Evolutionary stable strategies and game dynamics. Mathematical Biosciences 40 (1978) 145-156

5. Paolo, E.A.D., Noble, J., Bullock, S.: Simulation models as opaque thought experiments. In Bedau, M.A., McCaskill, J.S., Packard, N., Rasmussen, S., eds.: Proceedings of the Seventh International Conference on Artificial Life, Cambridge, MA, MIT Press (2000) 497-506

6. Nowak, M.A., May, R.M.: The spatial dilemmas of evolution. International Journal of Bifurcation and Chaos 3(1) (1993) 35-78

7. Pfeiffer, T., Schuster, S., Bonhoeffer, S.: Cooperation and competition in the evolution of ATP-producing pathways. Science 292(5516) (2001) 504-507

8. Pfeiffer, T., Bonhoeffer, S.: An evolutionary scenario for the transition to undifferentiated multicellularity. PNAS 100(3) (2003) 1095-1098

9. Kreft, J.U.: Biofilms promote altruism. Microbiology 150 (2004) 2751-2760

10. Bull, J.J., Molineux, I.J., Rice, W.R.: Selection of benevolence in a host-parasite system. Evolution 45(4) (1991) 875-882

11. Kerr, B., Neuhauser, C., Bohannan, B.J.M., Dean, A.M.: Local migration promotes competitive restraint in a host-pathogen 'tragedy of the commons'. Nature 442 (2006) $75-78$

12. Hardin, G.: The tragedy of the commons. Science 162 (1968) 1243-1248

13. Maynard Smith, J.: Evolution and the Theory of Games. Cambridge University Press (1982)

14. Rainey, P.B., Rainey, K.: Evolution of cooperation and conflict in experimental bacterial populations. Nature 425 (2003) 72-74

15. Killingback, T., Bieri, J., Flatt, T.: Evolution in group-structured populations can resolve the tragedy of the commons. Proceedings of the Royal Society B $\mathbf{2 7 3}$ (2006) 1477-1481

16. Ohtsuki, H., Hauert, C., Lieberman, E., Nowak, M.A.: A simple rule for the evolution of cooperation on graphs and social networks. Nature 441 (2006) 502505 